\title{
Dynamical Analysis and Simulation Validation of Incompletely Restrained Cable-Suspended Swinging System Driven by Two Cables
}

\author{
Naige Wang, ${ }^{1,2}$ Guohua Cao, ${ }^{1,2}$ Zhencai Zhu, $^{1,2}$ and Weihong Peng ${ }^{3}$ \\ ${ }^{1}$ School of Mechatronic Engineering, China University of Mining and Technology, Xuzhou 221116, China \\ ${ }^{2}$ Jiangsu Key Laboratory of Mine Mechanical and Electrical Equipment, China University of Mining and Technology, \\ Xuzhou 221116, China \\ ${ }^{3}$ School of Mechanics and Civil Engineering, China University of Mining and Technology, Xuzhou 221116, China
}

Correspondence should be addressed to Guohua Cao; caoguohua@cumt.edu.cn

Received 26 June 2016; Accepted 29 August 2016

Academic Editor: Mickaël Lallart

Copyright (C) 2016 Naige Wang et al. This is an open access article distributed under the Creative Commons Attribution License, which permits unrestricted use, distribution, and reproduction in any medium, provided the original work is properly cited.

\begin{abstract}
The flexibility of the suspension multicables and driven length difference between two cables cause the translation and rotation of the platform in the incompletely restrained cable-suspended system driven by two cables (IRCSWs2), which are theoretically investigated in this paper. The suspension cables are spatially discretized using the assumed modes method (AMM) and the equations of motion are derived from Lagrange equations of the first kind. Considering all the geometric matching conditions are approximately linear with external actuator, the differential algebraic equations (DAEs) are transformed to a system of ordinary differential equations (ODEs). Using linear boundary conditions of the suspension cable, the current method can obtain not only the accurate longitudinal displacements of cable and posture of the platform, but also the tension between the platform and cables, and the current method is verified by ADAMS simulation.
\end{abstract}

\section{Introduction}

The dynamics of cable-driven parallel mechanisms have been a topic of interest since the introduction of the first designs. Cable-driven parallel mechanisms, due to cable light weight and the ability to resist relatively large axial load, have a good choice for a variety of applications, such as telescope [1], haptic interface [2], elevators [3,4], rescue robotics [5], heavy load transportation [6], and mine hoisting [7]. Cable-driven parallel mechanisms are suitable for lifting, positioning large heavy load, and digging a vertical shaft.

The cable-driven mechanisms are generally divided into two categories: the full constrained cable-driven mechanism $[8,9]$ and cable-suspended mechanism [10-12]. The moving platform of the full constrained cable-driven mechanism can fully control all degrees of freedom; however, the suspended cable mechanism uses external force to maintain all cables in tension. Many cable-driven parallel mechanisms have been mostly considered quasi-static devices; for instance, classic determination method of the static workspace is addressed in the related literature [13]. Dynamic models of cablesuspended mechanism are built and studied on the optimal tension distribution among the cable [14] and the deflection of the suspended platform under the varying cable length. Dynamic behaviors of cable-suspended mechanism are also studied in $[15,16]$ and others.

All the works as mentioned above did not consider the influence of the longitudinal vibration of cable on cablesuspended mechanisms; however, they are subjected to the longitudinal vibration caused by the external excitation for high flexibility of cable. The longitudinal vibrations of cables have been studied extensively for decades. Particularly, in the typical sinking winch mechanism [17-19], simulation platforms of a ship and a portable crane, the mechanism used to sink the vertical shaft is composed of winches, head sheaves, a construction platform, and suspension cables. In this mechanism, the platform is hung by suspension cables. Deb [20] analyzed the lateral vibration of a string fixed at both 
ends excited by the lateral impact of an elastic load at any point using Heaviside's expansion theorem. Subsequently, many scholars have focused on the vibrations of cables characterized by time-varying length. Nguyen and Hong [21] analyzed the longitudinal and transverse vibrations of an axially moving string system at right side exerted by a hydraulic actuator equipped with a damper using Hamilton's principle. Sandilo and van Horssen [22] studied the free lateral responses of vertically translating media with variable length, velocity, and tension, subject to general initial conditions. The cable-suspended mechanism consists of not only distributed-parameter components, such as the suspension cable, but also lumped-parameter components, such as the head sheave and the suspended platform [23]. A reduction method is developed by Zhu et al. [24, 25] to deal with such structural system into distributed- and lumped-parameter components, and the distributed-parameter components can be discretized using the AMM and the complicated natural matching conditions can serve as the constraints of the generalized coordinates [26].

Indeed, cable-driven parallel mechanisms have the potential to produce very fast motions and the control of such motions requires a proper understanding of the vibration of the mechanical system [22, 27]. However, swing conditions often occur on these platforms. In order to improve reliability of equipment on these swing conditions, the swing experiments are designed for testing the behaviors of equipment and elevators. An important feature of the system is that the platform is shaken by the suspension cables. In addition, the tensions in the suspension cables should be properly allocated to meet the safety and performance requirements. Hence, it is necessary to evaluate the design by calculating the dynamic responses of the platform and the cables.

\section{Description of Incompletely Restrained Cable-Suspended Swinging System (IRCSWs2)}

According to the schematic, the 3D model of the novel IRCSWs2 is designed in Figure 1. The IRCSWs 2 is composed of two cables, pulleys, actuators, base frame, and suspended platform. Four connecting joints are symmetrically distributed around the suspended platform. Each cable is driven by an actuator in the middle of each cable, and it can be driven back and forth with a periodic motion. The system is equivalent to the suspended platform hung with four suspension cables.

For simplicity and without loss of generality, IRCSWs2 (Figure 1) can be described as the $i$ th suspension system model shown in Figures 2(a) and 2(b). Table 1 explains the meaning of the symbols adopted in Figure 2. This model is composed of four suspension cables of displacement $l_{i}(t)$ at time instant $t$ and the total length $L_{i}$; each suspension cable is passed over a set of head sheaves, and the suspended platform is attached to the lower ends of the four suspension cables shown in Figure 2(a). Based on invariability of suspension cable's length, it can be straightened in vertical line shown in Figure 2(b) and the corresponding sheave is located with
TABLE 1: Meaning of symbols in Figure 2.

\begin{tabular}{|c|c|}
\hline$O-X Y Z$ & $\begin{array}{l}\text { Cartesian reference frame with origin at the } \\
\text { centroid of base frame }\end{array}$ \\
\hline$O_{b}-x y z$ & $\begin{array}{c}\text { Cartesian reference frame with origin at the } \\
\text { centroid of base frame }\end{array}$ \\
\hline$\left(x_{i}, y_{i}, z_{i}\right)$ & $\begin{array}{l}\text { Coordinates of the lower ends with respect to } \\
\qquad O_{b}-x y z\end{array}$ \\
\hline$\left(X_{i}, Y_{i}, Z_{i}\right)$ & $\begin{array}{l}\text { Coordinates of the upper ends with respect to } \\
\qquad O-X Y Z\end{array}$ \\
\hline$l_{i j}$ & The displacement of the $j$ th sheave \\
\hline$m_{i j}$ & The equivalent mass of the $j$ th sheave \\
\hline
\end{tabular}

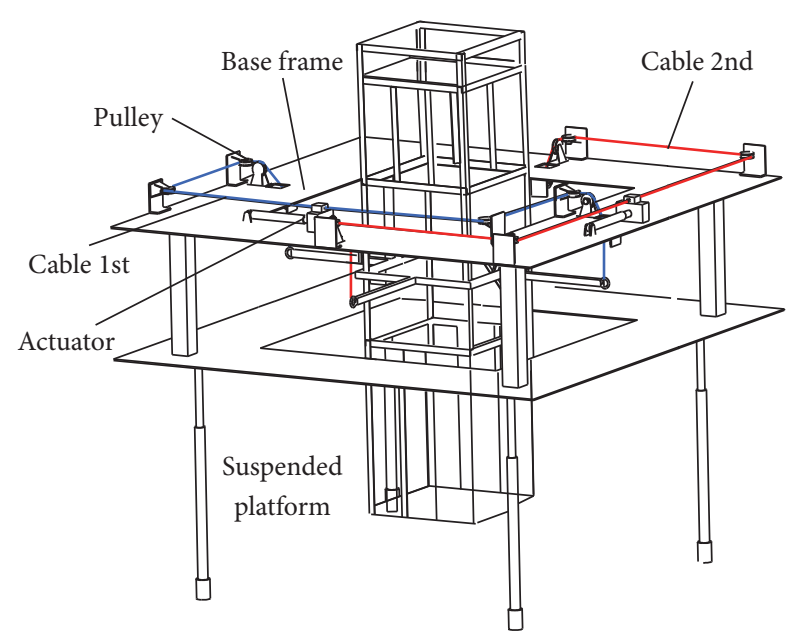

FIGURE 1: 3D model of IRCSWs2.

the same ratio of cable. To keep conversation of energy, elastic potential energy of the length $l_{i j}$ is only caused by constant value cable tension, and the rest cable $\left(L_{i}-l_{i j}\right)$ is caused by variable cable tension which is reflected in the total potential energy. In addition, the kinetic energy of sheaves is equivalent to concentrated masses on suspension cable which is reflected in its variable density. The mathematical model and natural characteristics of constant length of suspension cable considering the longitudinal vibration behavior and the concentrated mass of head sheave are proposed. The suspension cable has axial translating velocity $v_{i}(t)=\dot{l}_{i}(t)$ and acceleration $a_{i}(t)=\ddot{l}_{i}(t)$, where the overdot denotes time differentiation. It must be noted that the upper ends of suspensions cables are fixed to the actuator while the lower ends are attached to the suspended platform. Figures 2(a) and 2(b) describe the longitudinal vibrations along the axial transport motion. The displacement $u_{i}$ represents the longitudinal displacement of the particle of $i$ th suspension cable at position $x$ at time $t$.

The suspended platform is driven by four suspension cables with the upper ends fixed at actuators. It takes the actuator displacement as input and then takes the pose of the suspended platform and tensions and longitudinal vibration in cables as output. The input-output mathematics model of such a system is derived. When one actuator is working 


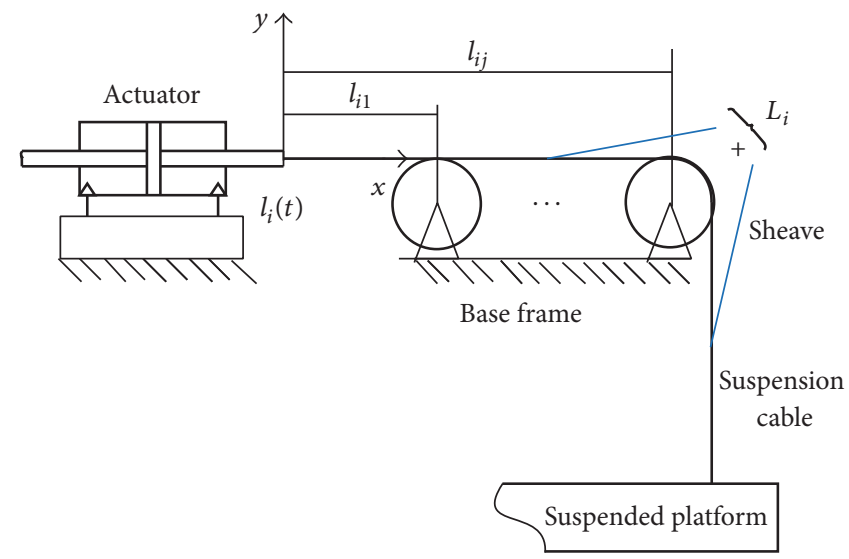

(a)

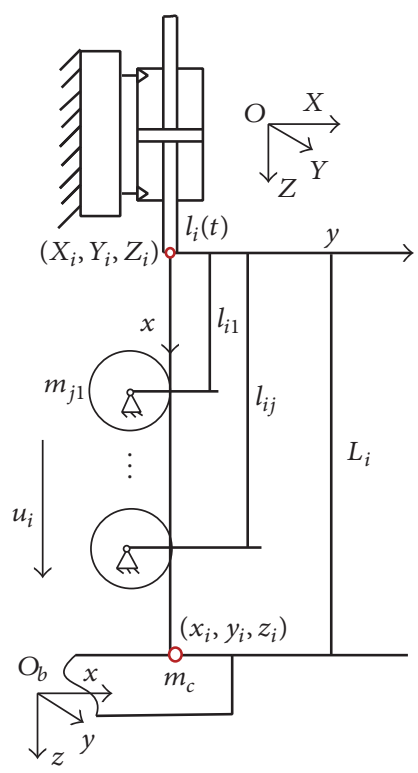

(b)

Figure 2: The $i$ th suspension system model.

independently, the suspended platform is swinging motion about a single coordinate axis.

In this paper, the AMM and Lagrange equations of the first kind are combined and applied to IRCSWs2. The suspension cables and the platform components are connected by mechanical joints that impose restrictions on their relative motion. Finite rotations introduce geometric nonlinearities; hence, the systems are inherently nonlinear. Mechanical joints result in algebraic constraints leading to a set of governing equations that combines differential and algebraic equations. Several challenging problems, such as the tensions between four suspension cables and the suspended platform, the deformations of the suspension cables, and the pose of the suspended platform, are treated. Four suspension cables are spatially discretized using the AMM and the equations of motion are derived from Lagrange equations of the first kind, while the geometric matching conditions at the interfaces of the cables are accounted for by the Lagrangian multiplier. The performance of dynamically periodic motions with small amplitude will be investigated. Thus, all the geometric matching conditions can be approximately linear, and the resulting spatially discretized equations, which are differential algebraic equations (DAEs), are transformed to ordinary differential equations (ODEs) and solved by an ODE solver.

\section{Theoretical Model of IRCSWs2}

3.1. Spatial Discretization. Ignoring the lateral vibration of the suspension cables and the influences of the frictional force, the IRCSWs2 can be modeled as four cables with fixed length and the pulleys can be set as lumped-parameter on cables. And the kinetic energy $K_{e}$ associated with the longitudinal vibrations is

$$
\begin{aligned}
K_{e}= & \sum_{i=1}^{4} \frac{1}{2} \int_{l_{i}(t)}^{L_{i}+l_{i}(t)} \rho_{i}(x)\left(\frac{D u_{i}}{D t}+v_{i}\right)^{2} \mathrm{~d} x \\
& +\left(\frac{1}{2} m_{c} \dot{\mathbf{r}}_{c}^{T} \cdot \dot{\mathbf{r}}_{c}+\frac{1}{2} \boldsymbol{\omega}^{T} \mathbf{I}_{c} \boldsymbol{\omega}\right),
\end{aligned}
$$

where $\rho_{i}(x)$ is the mass distribution function of the suspension cable and it is defined as $\rho_{i}(x)=\rho+\sum m_{i j} \delta\left(x-l_{i j}\right)$, $j=1 \sim n_{t}\left(n_{t}\right.$ is the number of the sheave of $i$ th suspension cable); $\rho$ and $\delta(\cdot)$, respectively, represent the unit mass and the Dirac delta function; $m_{c}$ and $\mathbf{I}_{c}$ are the mass and inertia tensor matrix of the suspended platform; $\mathbf{r}_{c}$ and $\boldsymbol{\omega}$ are the position and the angular velocity vectors of the suspended platform. The relationship of the body-fixed angular velocity vector $\boldsymbol{\omega}$ can be determined by resolving the Euler rates into the bodyfixed coordinate fame. The operator $D / D t$ is given by

$$
\frac{D}{D t}=\frac{\partial}{\partial t}+v_{i} \frac{\partial}{\partial x}
$$

Furthermore, the angular and linear velocity vectors of the suspended platform can be written as

$$
\begin{aligned}
\boldsymbol{\omega} & =\mathbf{E} \dot{\boldsymbol{\theta}}, \\
\dot{\boldsymbol{\theta}} & =\left[\begin{array}{lll}
\dot{\alpha} & \dot{\beta} & \dot{\gamma}
\end{array}\right]^{T}, \\
\dot{\mathbf{r}}_{c} & =\left[\begin{array}{lll}
\dot{x} & \dot{y} & \dot{z}
\end{array}\right]^{T},
\end{aligned}
$$


where

$$
\mathbf{E}=\left[\begin{array}{ccc}
\cos \beta \cos \gamma & \sin \gamma & 0 \\
-\cos \beta \sin \gamma & \cos \gamma & 0 \\
\sin \beta & 0 & 1
\end{array}\right]
$$

The inertia tensor of the platform with respect to the origin of $O_{b}-x y z$ is denoted as

$$
\mathbf{I}_{c}=\left[\begin{array}{ccc}
I_{x x} & I_{x y} & I_{x z} \\
I_{y x} & I_{y y} & I_{y z} \\
I_{z x} & I_{z y} & I_{z z}
\end{array}\right] .
$$

The total potential energy $V_{e}$ of all the cables is

$$
\begin{aligned}
V_{e}= & \sum_{i=1}^{4} \int_{l_{i}(t)}^{L_{i}+l_{i}(t)}\left[T_{i}(x, t) \varepsilon_{i}+\frac{1}{2} E A \varepsilon_{i}^{2}\right] \mathrm{d} x \\
& -\sum_{i=1}^{4} \int_{l_{i_{n_{t}}}(t)}^{L_{i}+l_{i}(t)} \rho_{i} g\left(u_{i}+x\right) \mathrm{d} x-m_{c} g z,
\end{aligned}
$$

where $E$ is Young's modulus of the cable; $A$ is the cross section area of cable; $g$ is gravity acceleration; $T_{i}(x, t)$ is the static tensions in the cables at position $x$ due to the gravitational acceleration $g$ and given as

$$
\begin{array}{r}
T_{i}(x, t)=T_{0 i}+\rho_{i} g\left(x-L_{i}-l_{i}(t) h\left(l_{i n_{t}}(t)-x\right)\right), \\
(i=1 \sim 4),
\end{array}
$$

where $T_{0 i}$ is the force of $i$ th suspension cable caused by the suspended platform and $h(x)$ is unit step function; if $x \geq 0$, then $h(x)=1$; else if $x<0$, then $h(x)=0$. The strain $\varepsilon_{i}$ can be approximated as $\varepsilon_{i}=\partial u_{i} / \partial x$. The first term in (8) represents the potential energy caused by the tension and strain in the suspension cable, while the second term represents the potential energy associated with gravity of the suspended platform and suspension cables.

The geometric boundary conditions for the suspension cable and the suspended platform are obtained as

$$
\begin{aligned}
g_{i} & =l_{i}(t)+L_{i}+u_{i}\left(l_{i}(t)+L_{i}, t\right)-\sqrt{\delta x^{2}+\delta y^{2}+\delta z^{2}} \\
& =0
\end{aligned}
$$

where

$$
\begin{aligned}
\delta x & =\left|\vartheta_{1,1}\right|, \\
\delta y & =\left|\vartheta_{2,1}\right|, \\
\delta z & =\left|\vartheta_{3,1}\right|, \\
\vartheta & =R\left[x_{i}, y_{i}, z_{i}\right]^{T}+\mathbf{r}_{c}-\left[X_{i}, Y_{i}, Z_{i}\right]^{T} \quad\left(\vartheta \in \mathbb{R}^{3 \times 1}\right),
\end{aligned}
$$

in which the rotation matrix $\mathbf{R}$ can be written in terms of the roll-pitch-yaw Euler angles as follows:

$$
\begin{aligned}
& \mathbf{R} \\
& =\left(\begin{array}{ccc}
c \beta \cdot c \gamma & -c \beta \cdot s \gamma & s \beta \\
c \alpha \cdot s \gamma+c \gamma \cdot s \alpha \cdot s \beta & c \alpha \cdot c \gamma-s \alpha \cdot s \beta \cdot s \gamma & -c \beta \cdot s \alpha \\
s \alpha \cdot s \gamma-c \alpha \cdot c \gamma \cdot s \beta & c \gamma \cdot s \alpha+c \alpha \cdot s \beta \cdot s \gamma & c \alpha \cdot c \beta
\end{array}\right),
\end{aligned}
$$

where $c(\cdot)$ and $s(\cdot)$ denote shorthand writings for $\sin (\cdot)$ and $\cos (\cdot)$ functions, respectively. And $\alpha, \beta$, and $\gamma$ are the rotation angles around the fixed axes $x, y$, and $z$, respectively.

The paper is focused on the influence of small cables' axial motion due to the longitudinal variation of cable length on their dynamic behaviors. With the linear vibration approximations, that is, $|\kappa| \ll 1, \sin \kappa \approx \kappa, \cos \kappa \approx 1,(\kappa=\alpha, \beta, \gamma)$, and second-order item omitted, the nonlinear geometric matching conditions at the interface between the suspension cable and the suspended platform are rewritten as

$$
\begin{aligned}
g_{i}= & L_{i}+u_{i}\left(l_{i}(t)+L_{i}, t\right) \\
& -\left(z-Z_{i}(t)+z_{i}-\beta x_{i}+\alpha y_{i}\right)=0, \quad i=1 \sim 4 .
\end{aligned}
$$

The transformation $\xi=\left(x-l_{i}(t)\right) / L_{i}$ is used to transform the time-varying spatial domain $\left[l_{i}(t), l_{i}(t)+L_{i}\right]$, which is converted to a fixed domain $[0,1]$ for $\xi$. By using the separation of variables method, the longitudinal displacements can be approximated by expansions of a complete set of trial functions and expressed as

$$
u_{i}(\xi, t)=\sum_{k=1}^{n} U_{i, k}(\xi) q_{i, k}(t),
$$

where $n$ represent the number of assumption mode methods; $q_{i, k}(t)$, in which $i=1 \sim 4$, are the generalized coordinates; $U_{i, k}$ are the trial functions and should satisfy the homogeneous boundary conditions in (13) and can be expressed as

$$
U_{i, k}(\xi)=\sqrt{2} \sin \left(\frac{2 k-1}{2} \pi \xi\right) .
$$

Substituting (1), (8), and (13) into Lagrange equations of the first kind

$$
\frac{\partial}{\partial t} \frac{\partial K_{e}}{\partial \dot{q}_{k}}-\frac{\partial K_{e}}{\partial q_{k}}+\frac{\partial V_{e}}{\partial q_{k}}=\sum_{i=1}^{4} \lambda_{i} \frac{\partial g_{i}}{\partial q_{k}}
$$

yields the equations of motion with holonomic constraints

$$
\begin{aligned}
\mathbf{M} \ddot{\mathbf{q}} & =\mathbf{Q}(\mathbf{q}, \dot{\mathbf{q}}, t)+\mathbf{G}^{T} \boldsymbol{\lambda}, \\
\mathbf{g}(\mathbf{q}, t) & =0,
\end{aligned}
$$

where $\mathbf{M}$ is mass matrices; $\mathbf{Q}$ is force vector; $\mathbf{q}=\left(\mathbf{q}_{1}^{T}, \ldots, \mathbf{q}_{4}^{T}\right.$, $\left.\mathbf{r}_{c}^{T}, \boldsymbol{\theta}^{T}\right)^{T}\left(\mathbf{q}_{i}=\left[q_{i, 1}, \ldots, q_{i, n}\right]^{T}, i=1 \sim 4\right)$ is the vector of generalized coordinates; and it should be noted that these $(4 n+$ 6) generalized coordinates are not independent; however, the geometric matching conditions (13) yield the holonomic constraints $\mathbf{g}$ of the generalized coordinates, where $\mathbf{g}=$ $\left(g_{1}, g_{2}, g_{3}, g_{4}\right)^{T}$ is a vector including all the constraint conditions in (13); $\mathbf{G}=\partial \mathbf{g} / \partial \mathbf{q}$ is the Jacobian of the constraint equations, which is a $4 \times(4 n+6)$ matrix; and $\lambda$ is the array of 4 Lagrange's multipliers; denote the tensions between the suspension cable and the suspended platform.

For selection of the independent generalized coordinates, a transformation matrix $\mathbf{T}_{r}$ is introduced,

$$
\mathbf{p}=\mathbf{T}_{r} \cdot \mathbf{q},
$$


where $\mathbf{p}=\left(q_{1,1}, \ldots, q_{4,1}, \ldots, q_{1, k}, \ldots, q_{4, k}, \ldots, q_{1, n}, \ldots, q_{4, n}\right.$, $\left.\mathbf{r}_{c}^{T}, \boldsymbol{\theta}^{T}\right)^{T}$ is another sequence of the generalized coordinates. The matrices $\mathbf{M}$ and $\mathbf{Q}$ are expressed as

$$
\begin{aligned}
& \mathbf{M}=\mathbf{T}_{r} \overline{\mathbf{M}} \mathbf{T}_{r}^{T}, \\
& \mathbf{Q}=\mathbf{T}_{r} \overline{\mathbf{Q}},
\end{aligned}
$$

where

$$
\begin{aligned}
& \overline{\mathbf{M}}=\operatorname{diag}\left(\overline{\mathbf{M}}^{1}, \overline{\mathbf{M}}^{2}, \overline{\mathbf{M}}^{3}, \overline{\mathbf{M}}^{4}, \mathbf{M}_{p}\right) \\
& \overline{\mathbf{Q}}_{i}=\overline{\mathbf{F}}^{i}-\overline{\mathbf{C}}^{i} \dot{\mathbf{q}}_{i}-\overline{\mathbf{K}}^{i} \mathbf{q}_{i}, \\
& \overline{\mathbf{Q}}=\left[\overline{\mathbf{Q}}_{1}^{T}, \overline{\mathbf{Q}}_{2}^{T}, \overline{\mathbf{Q}}_{3}^{T}, \overline{\mathbf{Q}}_{4}^{T}, \overline{\mathbf{Q}}_{p}^{T}\right]^{T} \\
& \bar{M}_{i j}=L_{i} \int_{0}^{1} \rho_{i} U_{i}(\xi) U_{j}(\xi) \mathrm{d} \xi \\
& \bar{C}_{i j} \\
& =v_{i}\left(\int_{0}^{1} \rho_{i} U_{i}^{\prime}(\xi) U_{j}(\xi) \mathrm{d} \xi-\int_{0}^{1} \rho_{i} U_{i}(\xi) U_{j}^{\prime}(\xi) \mathrm{d} \xi\right) \\
& \bar{K}_{i j} \\
& =a_{i} \int_{0}^{1} \rho_{i} U_{i}^{\prime}(\xi) U_{j}(\xi) \mathrm{d} \xi \\
& +\frac{1}{L_{i}} \int_{0}^{1}\left(E A-\rho_{i} v^{2}\right) U_{i}^{\prime}(\xi) U_{j}^{\prime}(\xi) \mathrm{d} \xi \\
& \bar{F}_{i} \\
& =-L_{i} \int_{0}^{1} \rho_{i}\left(a_{i}-g\right) U_{j}(\xi) \mathrm{d} \xi+v_{i}^{2} \int_{0}^{1} \rho_{i} U_{j}^{\prime}(\xi) \mathrm{d} \xi \\
& -\int_{0}^{1} T_{i}(x, t) U_{j}^{\prime}(\xi) \mathrm{d} \xi \\
& -L_{i} \mathcal{g} \int_{0}^{l_{i n_{t}}(t) /\left(L_{i}+l_{i}(t)\right)} \rho_{i}(\xi) U_{j}(\xi) \mathrm{d} \xi \\
& \mathbf{M}_{p}=\left[\begin{array}{ll}
m_{c} \mathbf{I}_{3} & \\
& \mathbf{R}^{T} \mathbf{I}_{c} \mathbf{R}
\end{array}\right] \\
& \overline{\mathbf{Q}}_{p}=\left[\overline{\mathbf{Q}}_{p}^{1}, \overline{\mathbf{Q}}_{p}^{2}\right]^{T} \text {, } \\
& \overline{\mathbf{Q}}_{p}^{1}=\left[\begin{array}{lll}
0 & 0 & m_{c} g z
\end{array}\right] \\
& \overline{\mathbf{Q}}_{p}^{2} \\
& =\frac{1}{2} \dot{\boldsymbol{\theta}}^{T}\left[0, \frac{\partial \mathbf{E}^{T}}{\partial \beta} \mathbf{I}_{c} \mathbf{E}+\mathbf{E}^{T} \mathbf{I}_{c} \frac{\partial \mathbf{E}}{\partial \beta}, \frac{\partial \mathbf{E}^{T}}{\partial \gamma} \mathbf{I}_{c} \mathbf{E}+\mathbf{E}^{T} \mathbf{I}_{c} \frac{\partial \mathbf{E}}{\partial \gamma}\right] \dot{\boldsymbol{\theta}} \\
& -\left[\left(\frac{\partial \mathbf{E}^{T}}{\partial t} \mathbf{I}_{c} \mathbf{E}+\mathbf{E}^{T} \mathbf{I}_{c} \frac{\partial \mathbf{E}}{\partial t}\right) \dot{\boldsymbol{\theta}}\right]^{T} .
\end{aligned}
$$

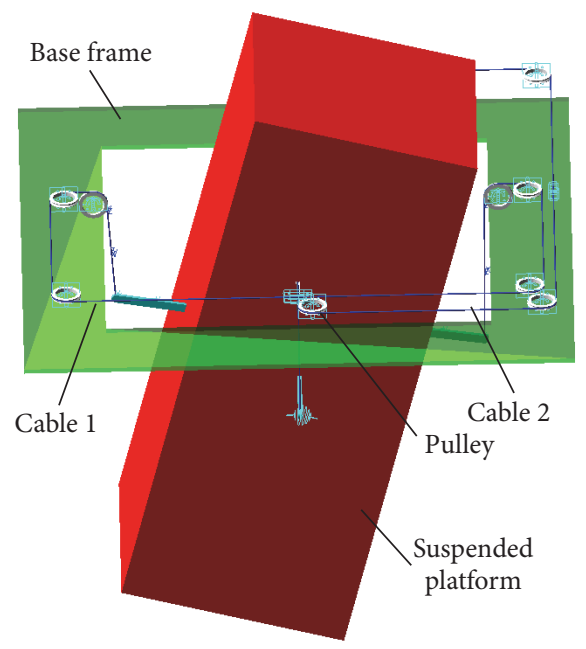

Figure 3: ADAMS simulation model.

3.2. DAEs to ODEs for Solution. Considering the constraint conditions in (13) are linear algebraic equations (17), a system of DAEs could be transformed to ODEs. Equation (17) could be expressed in the form

$$
\mathbf{g}(\mathbf{p}, t)=\mathbf{G} \mathbf{p}+\mathbf{g}_{r}(t)=\mathbf{0}
$$

By reduction method, the independent generalized coordinate vector $\mathbf{p}_{1}$ is selected and the DAEs of constrained mechanical system can be transformed to ODEs problem. Thus the cable tensions are obtained by evaluating (23)

$$
\begin{aligned}
& \boldsymbol{\Phi}^{T} \mathbf{M} \Phi \ddot{\mathbf{p}}_{1}=-\boldsymbol{\Phi}^{T} \mathbf{M} \ddot{\mathbf{u}}(t)+\boldsymbol{\Phi}^{T} \mathbf{Q}\left(\mathbf{p}_{1}, \dot{\mathbf{p}}_{1}, t\right) \\
& \left(\mathbf{T}_{r} \mathbf{G}^{T}\right)^{-1}\left(\boldsymbol{\Phi}^{T} \mathbf{M} \boldsymbol{\Phi}\left(\mathbf{U} \ddot{\mathbf{p}}_{1}+\ddot{\mathbf{u}}(t)\right)-\mathbf{Q}\left(\mathbf{p}_{1}, \dot{\mathbf{p}}_{1}, t\right)\right) \\
& \quad=\lambda
\end{aligned}
$$

The parameters $\mathbf{G}, \mathbf{g}_{r}(t), \ddot{\mathbf{p}}_{1}, \dot{\mathbf{p}}_{1}, \boldsymbol{\Phi}$, and $\ddot{\mathbf{u}}(t)$ in $(21)$ and (22) are shown in Appendix. And the initial displacement and velocity of the suspension cable are necessary for the numerical solution using Runge-Kutta method.

\section{Simulation Model of IRCSWs2}

An ADAMS simulation is built in order to validate the performance, which is shown in Figure 3. The method and procedure are presented by referring to literature [28]. The key parameters involved in the dynamic simulation of IRCSW 2 are listed in Notations.

The distance between the cable attachment points to the origin of $\mathrm{O}_{b}-x y z$ is $2.07 \mathrm{~m}$. Two translational actuators are built to control the suspension cable with periodic motion. The actuator movement profile is shown in Figure 4. The actuator displacement is sine function with amplitude $0.2 \mathrm{~m}$ and frequency $0.1 \mathrm{~Hz}$, while the jerk is constant during startup. The equivalent mass of sheaves is assumed to be constant. 


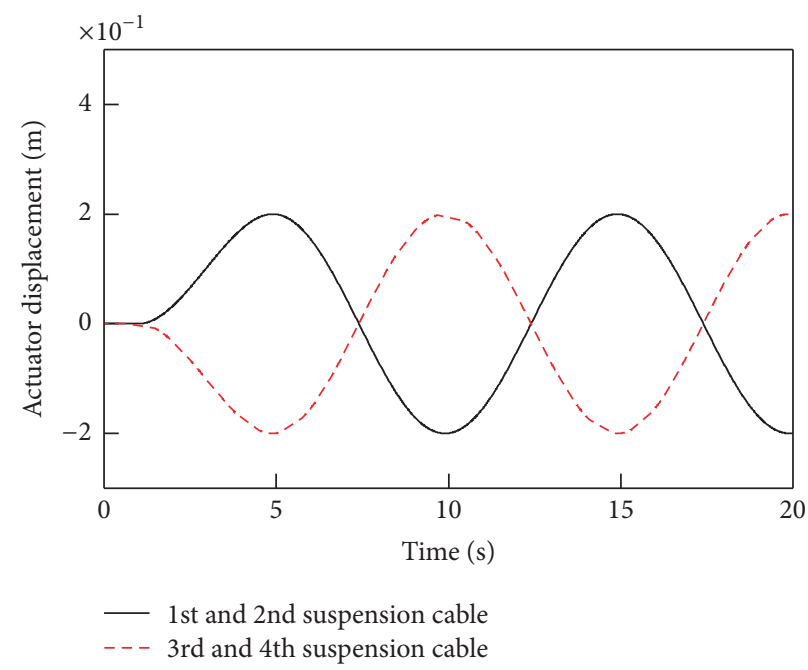

(a)

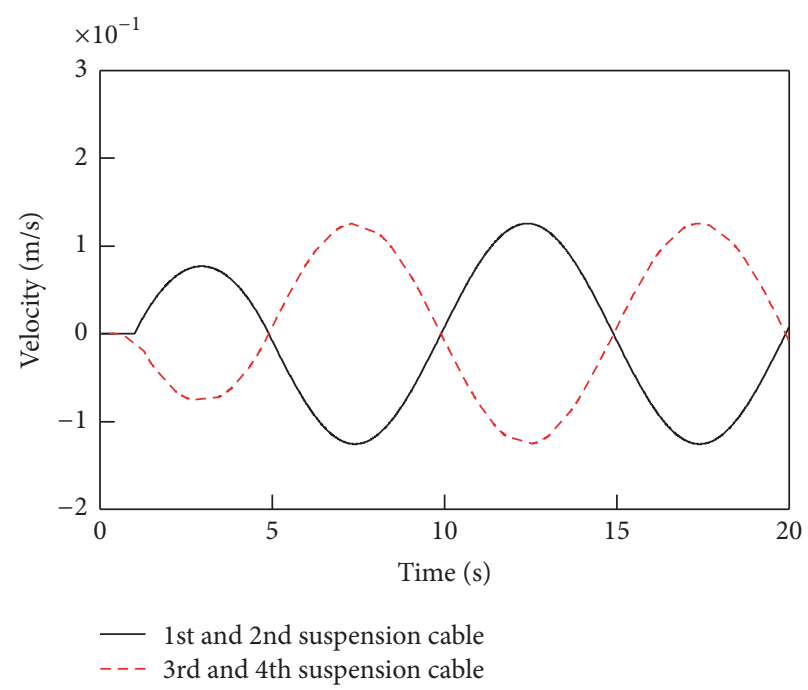

(b)

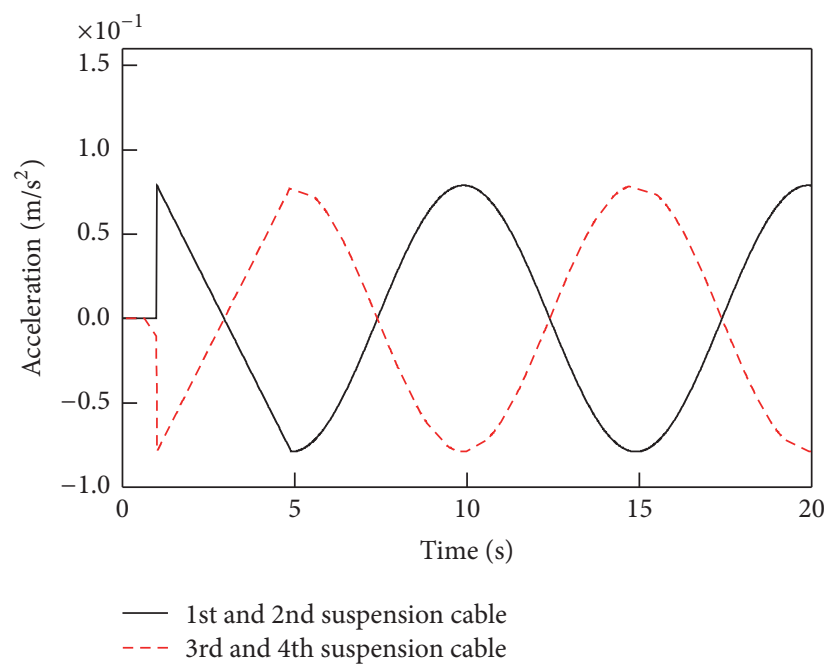

(c)

FIGURE 4: Movement profiles: (a) displacement $l_{i}(t)$; (b) velocity $v_{i}(t)$; (c) acceleration $a_{i}(t)$.

4.1. The Convergence Analysis of IRCSWs2. It is necessary to know the number of terms about assumption mode method which decides the reliability of the result and computation time. Above all, the convergence of the solution for governing equation is examined by varying the number of included modes in Figure 5. The results indicate that the model $(n=$ $10)$ converges very fast under lower order modes. In order to decrease computing time, the number of the trial function used in the AMM is $n=10$.

4.2. Examples and Discussion. The results of theoretical calculation and ADAMS simulation are denoted by black lines and red lines in Figures 6, 7, and 8, respectively. Two translation joints are used to control the status of the cables. Swing motions were successfully demonstrated with the results. All results present approximately sinusoidal variation with a sinusoidal excitation in Figure 6 . The axial tension has finite impulse response that is caused by sudden switch at 1 second just as shown in Figure 7. The displacements along the $x$ axis, $y$ axis and $z$ axis are nearly zero, which validated the assumptions under small excitation.

The two results of theoretical calculation and ADAMS simulation are in excellent agreement (Figure 7). The cable tension ranges between $28 \mathrm{kN}$ and $33 \mathrm{kN}$ which indicates that the cable tension is small compared to the tensile capacity of the cable (safety factor larger than 4) and that auxiliary protections are usually designed in the practice to prevent failures. The results show that the longitudinal vibration is only slightly smaller than ADAMS results due to neglecting the rigid body displacement of the suspended platform in $x$ and $y$ direction (Figures 6 and 8); however, during a cycle time of $10 \mathrm{~s}$, their variable trends have no great difference. The higher mode of high-frequency variation is caused by the constant jerk at the beginning of the motion. Due to the low driving frequency and damping effect, this highfrequency variation is quick to disappear. Therefore, the 


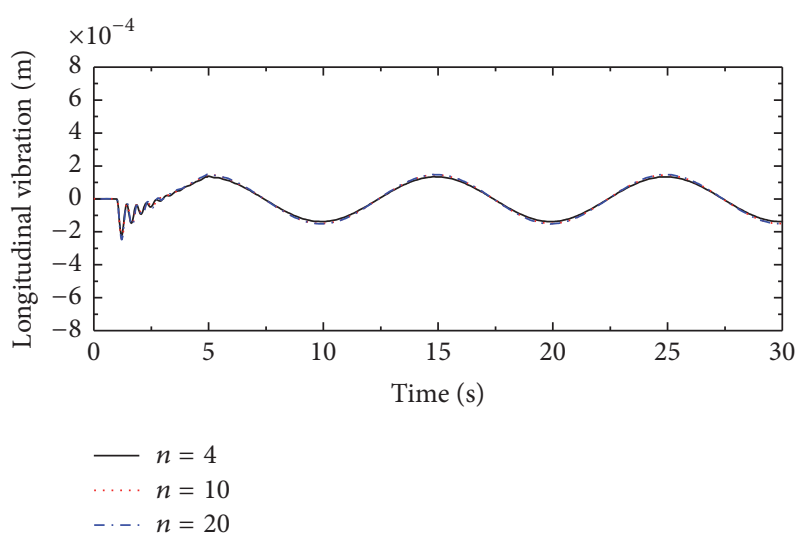

FIgURE 5: Longitudinal response with different numbers of modes.

theoretical model and the ADAMS simulation model are both reasonable, and these two models could be adopted independently to study the behavior of IRCSWs2.

For the normal case of system resonance and low levels of excitation, the phase trajectory of the generalized coordinate $q_{11}$ when the initial stage is zeros is shown in Figure 9. Circle family of phase trajectory is changing when the excitation is different. The status of the system accelerations over an extended period and the system behavior are on steady state of maintaining swing motion of suspended platform with system dissipation. In the upper half plane $\left(\mathrm{d} q_{11}>0\right)$, the motion direction of system status is along positive direction of horizontal axis; in the down half plane $\left(\mathrm{d} q_{11}<0\right)$, the phase trajectory moves to left similarity. The rate of phase trajectory at horizontal axis is always parallel to the vertical axis of the graph.

\section{Conclusions}

The dynamical characteristic of IRCSWs2 is investigated in aspects of both theoretical model and the ADAMS simulation model. The following conclusions could be drawn:

(1) The focus of this paper is to introduce and analyze the motion of this novel IRCSWs2 by the AMM and Lagrange equations of the first kind. The performance of dynamically periodic motions with small amplitude is investigated. Thus, all the geometric matching conditions can be approximately linear, and the resulting spatially discretized equations are transformed to ordinary differential equations (ODEs) by reduction method.

(2) In order to validate the theoretical model, the corresponding ADAMS simulation is built. The results of theoretical model are consistent with the ADAMS simulation by comparison.

(3) The translation displacement of suspended platform is almost near zero, and rotations of angles about, respectively, $x$ - and $y$-axes present sinusoidal variation. The rotation about $z$ axes is tiny enough, which can be neglected. Tensions and longitudinal displacement also present sinusoidal variation, which is useful to design and optimize the cable-suspended system.

\section{Appendix}

Equation (13) could be expressed in the form

$$
\mathbf{g}(\mathbf{p}, t)=\mathbf{G} \mathbf{p}+\mathbf{g}_{r}(t)=\mathbf{0},
$$

where $\mathbf{g}_{r}(t)=\left[l_{1}(t), \ldots, l_{4}(t)\right]^{T}$. Without loss of generality, suppose

$$
\begin{aligned}
\mathbf{G} & =\left(\begin{array}{ll}
\mathbf{G}_{0} & \mathbf{G}_{1}
\end{array}\right), \\
\mathbf{p} & =\left[\begin{array}{ll}
\mathbf{p}_{0}^{T} & \mathbf{p}_{1}^{T}
\end{array}\right]^{T},
\end{aligned}
$$

where $\mathbf{G}_{0}$ must be a nonsingular $4 \times 4$ matrix, because its inverse matrix will be used subsequently. By (14) and (A.1) and (A.2), one can obtain

$$
\begin{aligned}
& \mathbf{G}_{0}=\operatorname{diag}\left(U_{1,1}(1), U_{2,1}(1), U_{3,1}(1), U_{4,1}(1)\right), \\
& \mathbf{G}_{1}=\left[\mathbf{G}_{2}^{c}, \ldots, \mathbf{G}_{n}^{c}, \mathbf{G}^{p}\right], \\
& \mathbf{p}_{0}=\left[p_{1,1}, p_{2,1}, p_{3,1}, p_{4,1}\right]^{T}, \\
& \mathbf{p}_{1}=\left(p_{1,2}, \ldots, p_{4,2}, \ldots, p_{1, j}, \ldots, p_{4, j}, \ldots, p_{1, n}, \ldots, p_{4, n}, \mathbf{r}_{c}^{T},\right. \\
&\left.\boldsymbol{\theta}^{T}\right)^{T},
\end{aligned}
$$

in which

$$
\begin{aligned}
\mathbf{G}_{j}^{c} & =\operatorname{diag}\left(U_{1, j}(1), U_{2, j}(1), U_{3, j}(1), U_{4, j}(1)\right), \\
\mathbf{G}^{p} & =\left[\mathbf{G}_{1}^{p}, \mathbf{G}_{2}^{p}, \mathbf{G}_{3}^{p}, \mathbf{G}_{4}^{p}\right]^{T}, \\
\mathbf{G}_{i}^{p} & =\left[0,0,1, y_{i},-x_{i}, 0\right] .
\end{aligned}
$$

G is constant matrix and can be accurately calculated. Comparing the vectors $\mathbf{p}$ and $\mathbf{q}$, if $\mathbf{q}$ is selected in (17), then $\mathbf{G}_{0}$ will be a singular matrix. By this method, the redundant constraints are eliminated and the DAEs of constrained mechanical system can be transformed to ODEs problem.

By substituting (A.2) into (A.1), one has

$$
\begin{aligned}
\mathbf{p} & =\boldsymbol{\Phi}(t) \mathbf{p}_{1}+\mathbf{u}(t), \\
\boldsymbol{\Phi}(t) & =\left[\begin{array}{c}
-\mathbf{G}_{0}^{-1} \mathbf{G}_{1} \\
\mathbf{I}
\end{array}\right], \\
\mathbf{u}(t) & =\left[\begin{array}{c}
-\mathbf{G}_{0}^{-1} \mathbf{g}_{r} \\
\mathbf{0}
\end{array}\right],
\end{aligned}
$$

where $\mathbf{p}_{1}$, a $(4 n+2)$ vector, become the reduced generalized coordinates, which are linearly independent; $\mathbf{I}$ is a $(4 n+2)$ identity matrix.

Differentiating (A.5) yields

$$
\begin{aligned}
& \dot{\mathbf{p}}=\boldsymbol{\Phi}(t) \dot{\mathbf{p}}_{1}+\dot{\boldsymbol{\Phi}}(t) \mathbf{p}_{1}+\dot{\mathbf{u}}(t), \\
& \ddot{\mathbf{p}}=\boldsymbol{\Phi}(t) \ddot{\mathbf{p}}_{1}+2 \dot{\boldsymbol{\Phi}}(t) \dot{\mathbf{p}}_{1}+\ddot{\boldsymbol{\Phi}}(t) \mathbf{p}_{1}+\ddot{\mathbf{u}}(t) .
\end{aligned}
$$



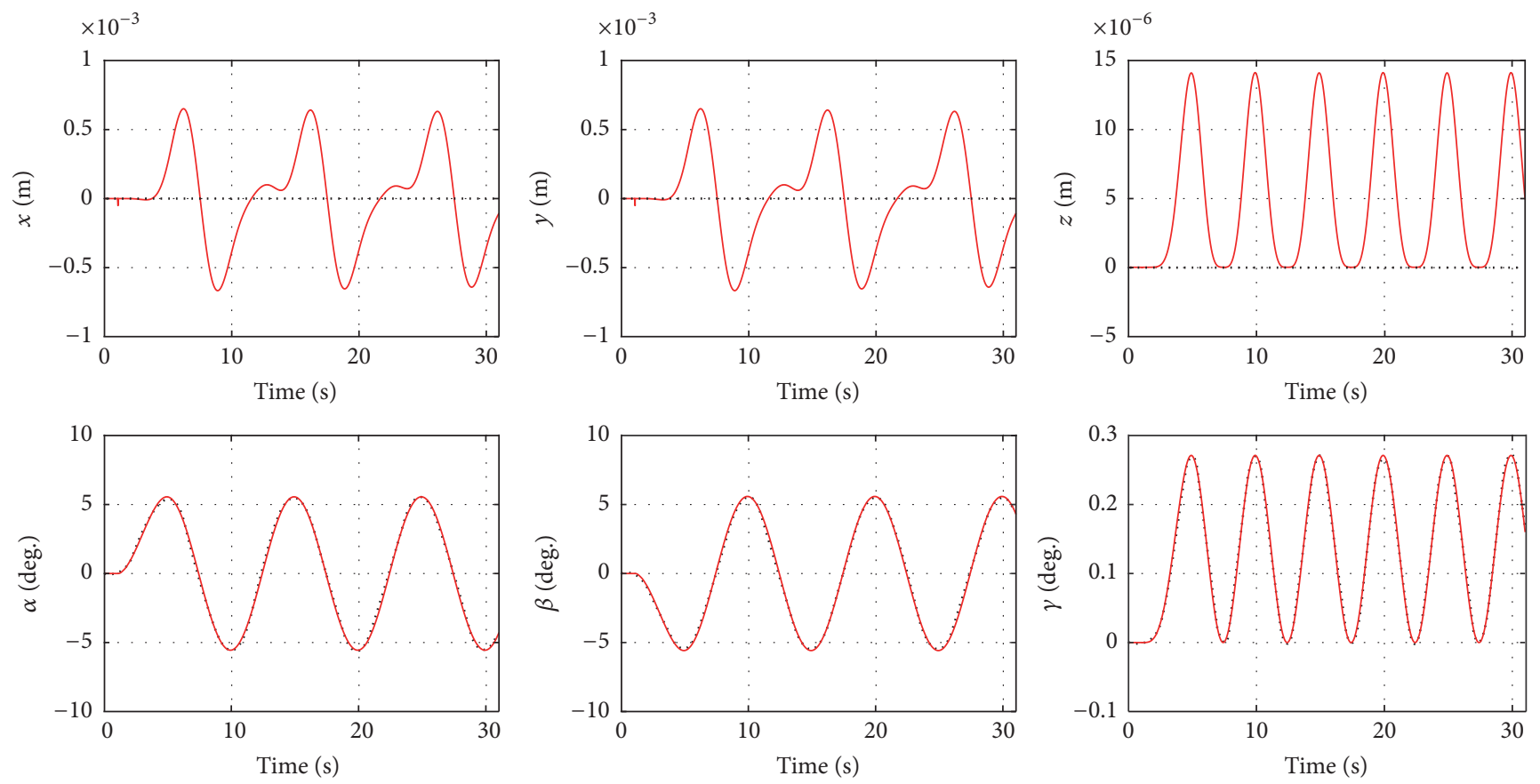

..... Theoretical model

..... Theoretical model

..... Theoretical model

- ADAMS model

— ADAMS model

FIgURE 6: The pose of the suspended platform.
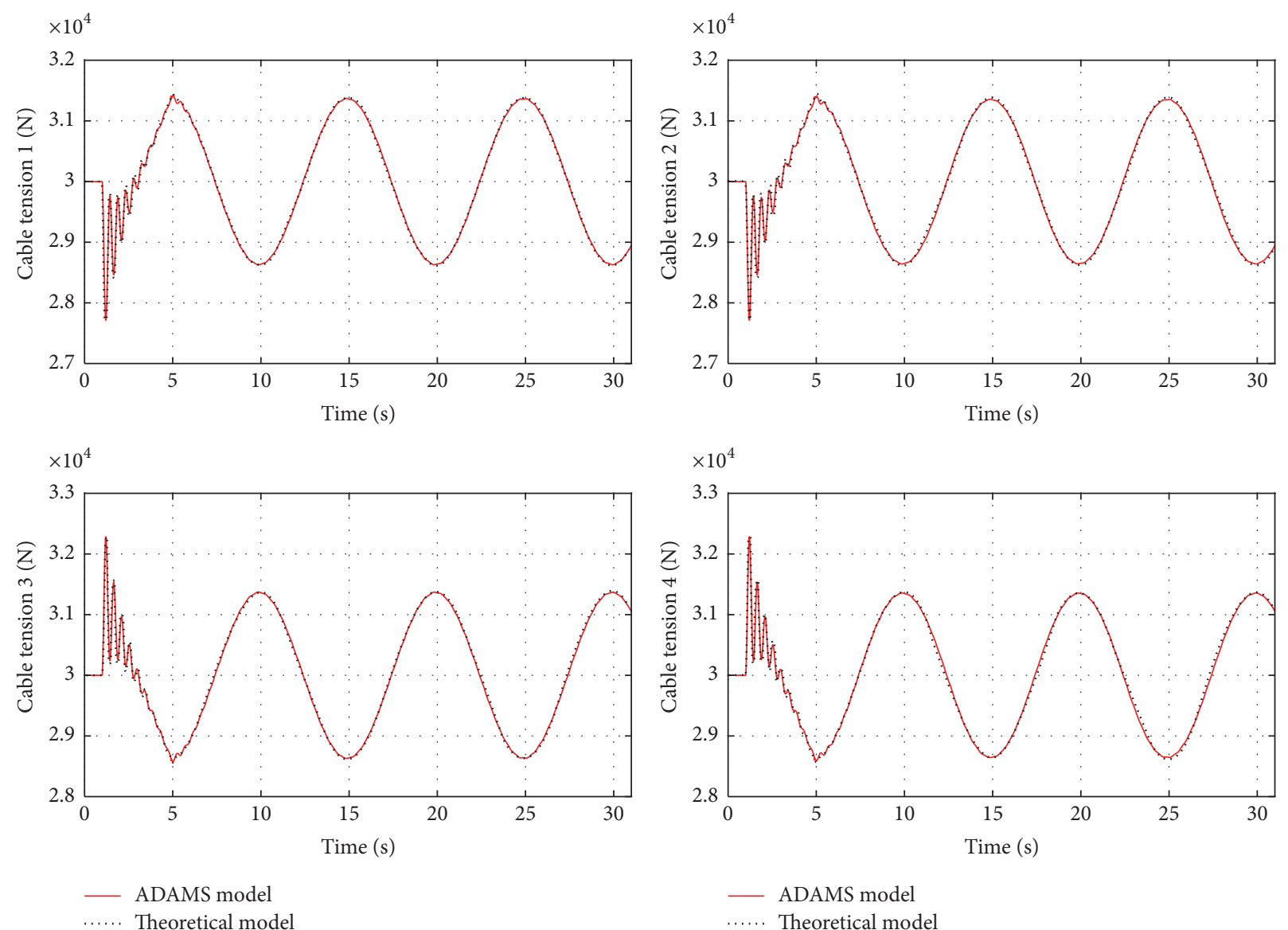

Figure 7: Cable tensions. 

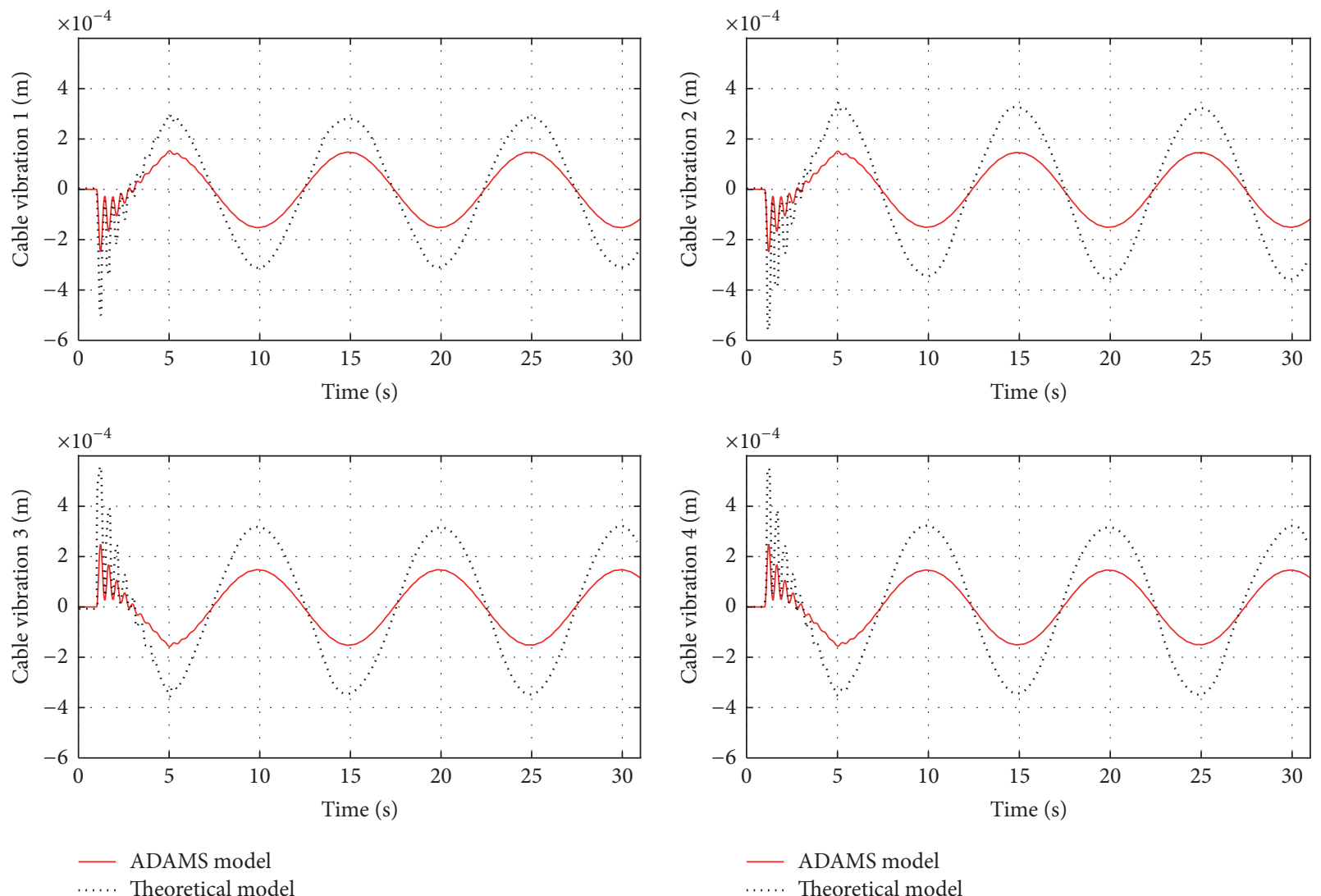

FIGURE 8: Longitudinal vibration.

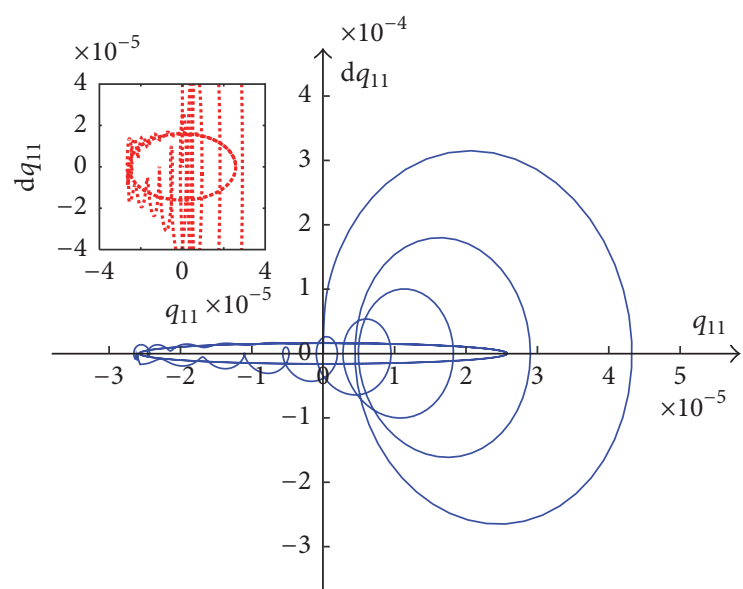

(a)

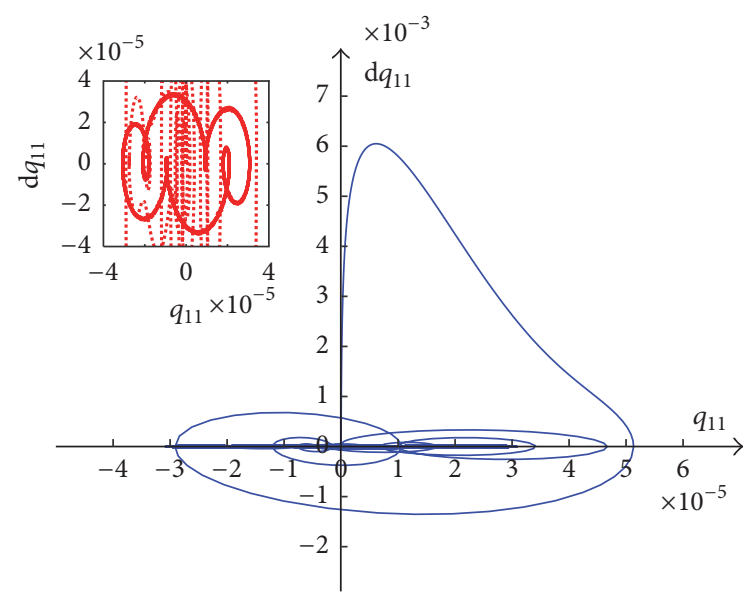

(b)

FIGURE 9: The phase trajectory of $q_{11}$ : (a) no longitudinal excitation; (b) the excitation of the four suspension cables is given by $e(t)=$ $0.01 \sin (\pi t)$.

Combining (A.2) and (A.5) can yield $\mathbf{G} \boldsymbol{\Phi}=\mathbf{0}$. Substituting (A.6) into the first equation in (17), premultiplying by $\boldsymbol{\Phi}^{T}$ and using the relations $\boldsymbol{\Phi}^{T} \mathbf{G}^{T}=(\mathbf{G} \boldsymbol{\Phi})^{T}=\mathbf{0}$ yield

$$
\mathbf{U}^{T} \mathbf{M U} \ddot{\mathbf{p}}_{1}=-\mathbf{U}^{T} \mathbf{M} \ddot{\mathbf{u}}(t)+\mathbf{U}^{T} \mathbf{Q}\left(\mathbf{p}_{1}, \dot{\mathbf{p}}_{1}, t\right),
$$

where $\mathbf{p}$ can be obtained by substituting the solution $\mathbf{p}_{1}$ into the geometric constraint equation (A.5) and $\mathbf{q}$ can be assembled as

$$
\mathbf{q}=\mathbf{T}_{r}\left(\Phi(t) \mathbf{p}_{1}+\mathbf{u}(t)\right)
$$


The cable is assumed to be at rest initially; hence the initial conditions for generalized coordinates are expressed as (A.9)

$$
\begin{aligned}
q_{i, k}(0)= & \int_{0}^{1} u_{i}\left(\xi L_{i}, 0\right) U_{i, k}(\xi) \mathrm{d} \xi \\
\dot{q}_{i, k}(0)= & \int_{0}^{1} u_{i}\left(\xi L_{i}, 0\right) U_{i, k}(\xi) \mathrm{d} \xi \\
& +v_{i}(0) \sum_{j=1}^{n} q_{i, j}(0) \int_{0}^{1} \xi U_{i, j}^{\prime}(\xi) U_{i, k}(\xi) \mathrm{d} \xi \\
& +\frac{v_{i}(0) \sqrt{l_{i}(0)}}{2} q_{i, j}(0) .
\end{aligned}
$$

\section{Notations}

\section{Key Parameters}

$\begin{array}{ll}\rho: & \begin{array}{l}\text { Mass per unit length of suspension cable } \\ (0.9753 \mathrm{~kg} / \mathrm{m})\end{array} \\ L_{i}: & \begin{array}{l}\text { Total length of each suspension cable } \\ (7.19 \mathrm{~m})\end{array} \\ m_{i j}: & \text { Equivalent mass of sheave }(10 \mathrm{~kg}) \\ E: & \text { Young's modulus }\left(1.2 \times 10^{5} \mathrm{MPa}\right) \\ g: & \text { Gravity Acceleration }\left(10 \mathrm{~m} / \mathrm{s}^{2}\right) \\ A: & \text { Cross section area of cable }\left(2.01 \times 10^{-4} \mathrm{~m}^{2}\right) \\ m_{c}: & \text { Mass of suspended platform }\left(1.2 \times 10^{4} \mathrm{~kg}\right) \\ f: & \text { The frequency of sinusoidal excitation } \\ & (0.1 \mathrm{~Hz}) \\ l_{i j}(j=1 \sim 3): & \text { The initial displacement of sheave }((2,4.8, \\ & 5.42) \mathrm{m}) \\ \mathbf{I}_{c}: & \text { Inertia tensor } \\ & \left(\text { diag }(1.48,1.48,0.08) \times 10^{5} \mathrm{~kg} \mathrm{~m}^{2}\right) .\end{array}$

\section{Competing Interests}

The authors declare that they have no competing interests.

\section{Acknowledgments}

This work is supported by the National Natural Science Foundation of China (51475456), the Fundamental Research Funds for the Central Universities (Grant no. 2014YC06), and the Priority Academic Program Development of Jiangsu Higher Education Institutions (PAPD).

\section{References}

[1] Y. X. Su, B. Y. Duan, R. D. Nan, and B. Peng, "Development of a large parallel-cable manipulator for the feed-supporting system of a next-generation large radio telescope," Journal of Robotic Systems, vol. 18, no. 11, pp. 633-643, 2001.

[2] A. Frisoli, F. Salsedo, and M. Bergamasco, "Design of a new tendon driven haptic interface with six degrees of freedom," in Proceedings of the 8th IEEE International Workshop on Robot and Human Interaction (RO-MAN '99), pp. 303-308, IEEE, Piscataway, NJ, USA, 1999.
[3] H. Taplak, S. Erkaya, Ş. Yildirim, and I. Uzmay, "The use of neural network predictors for analyzing the elevator vibrations," Arabian Journal for Science and Engineering, vol. 39, no. 2, pp. 1157-1170, 2014.

[4] L.-Q. Shi, Y.-Z. Liu, S.-Y. Jin, and Z.-M. Cao, "Numerical simulation of unsteady turbulent flow induced by two-dimensional elevator car and counter weight system," Journal of Hydrodynamics, vol. 19, no. 6, pp. 720-725, 2007.

[5] P. Bosscher, R. L. Williams, and M. Tummino, "A concept for rapidly-deployable cable robot search and rescue systems," in Proceedings of the 2005 ASME International Design Engineering Technical Conferences, Mechanics and Robotics Conference, pp. 589-598, American Society of Mechanical Engineers, Long Beach, Calif, USA, 2005.

[6] S. Lahouar, E. Ottaviano, S. Zeghoul, L. Romdhane, and M. Ceccarelli, "Collision free path-planning for cable-driven parallel robots," Journal of the Autonomic Nervous System, vol. 57, pp. 1083-1093, 2009.

[7] X.-G. Shao, Z.-C. Zhu, Q.-G. Wang, P. C. Chen, B. Zi, and G.-H. Cao, "Non-smooth dynamical analysis and experimental validation of the cable-suspended parallel manipulator," Proceedings of the Institution of Mechanical Engineers C, vol. 226, no. 10, pp. 2456-2466, 2012.

[8] A. Trevisani, P. Gallina, and R. L. Williams III, "Cable-directdriven robot (CDDR) with passive SCARA support: theory and simulation," International Journal of Advanced Robotic Systems, vol. 46, no. 1, pp. 73-94, 2006.

[9] M. A. Khosravi and H. D. Taghirad, "Robust PID control of fully-constrained cable driven parallel robots," Mechatronics, vol. 24, no. 2, pp. 87-97, 2014.

[10] S. Krut, N. Ramdani, M. Gouttefarde, O. Company, and F. Pierrot, "A parallel cable-driven crane for scara-motions," in Proceedings of the ASME International Design Engineering Technical Conferences and Computers and Information in Engineering Conference, pp. 101-108, American Society of Mechanical Engineers, Brooklyn, NY, USA, August 2008.

[11] K. Kozak, Q. Zhou, and J. S. Wang, "Static analysis of cabledriven manipulators with non-negligible cable mass," IEEE Transactions on Robotics, vol. 22, no. 3, pp. 425-433, 2006.

[12] M. H. Korayem and M. Bamdad, "Dynamic load-carrying capacity of cable-suspended parallel manipulators," International Journal of Advanced Manufacturing Technology, vol. 44, no. 7-8, pp. 829-840, 2009.

[13] S. Behzadipour and A. Khajepour, "Stiffness of cable-based parallel manipulators with application to stability analysis," Journal of Mechanical Design, vol. 128, no. 1, pp. 303-310, 2006.

[14] C. Gosselin and M. Grenier, "On the determination of the force distribution in overconstrained cable-driven parallel mechanisms," Meccanica, vol. 46, no. 1, pp. 3-15, 2011.

[15] C. Gosselin and S. Foucault, "Experimental determination of the accuracy of a three-DOF cable-suspended parallel robot performing dynamic trajectories," in Cable-Driven Parallel Robots: Proceedings of the Second International Conference on Cable-Driven Parallel Robots, vol. 32 of Mechanisms and Machine Science, pp. 101-112, Springer, Berlin, Germany, 2015.

[16] T. Bruckmann and A. Pott, Cable-Driven Parallel Robots, Springer, Berlin, Germany, 2013.

[17] S. Kaczmarczyk and W. Ostachowicz, "Transient vibration phenomena in deep mine hoisting cables. Part 2: numerical simulation of the dynamic response," Journal of Sound and Vibration, vol. 262, no. 2, pp. 245-289, 2003. 
[18] S. Kaczmarczyk and W. Ostachowicz, "Transient vibration phenomena in deep mine hoisting cables. Part 1: mathematical model," Journal of Sound and Vibration, vol. 262, no. 2, pp. 219244, 2003.

[19] S. Kaczmarczyk, "The passage through resonance in a catenaryvertical cable hoisting system with slowly varying length," Journal of Sound and Vibration, vol. 208, no. 2, pp. 243-269, 1997.

[20] K. K. Deb, "Dynamics of a string and an elastic hammer," Journal of Sound and Vibration, vol. 40, no. 2, pp. 243-248, 1975.

[21] Q. C. Nguyen and K.-S. Hong, "Simultaneous control of longitudinal and transverse vibrations of an axially moving string with velocity tracking," Journal of Sound and Vibration, vol. 331, no. 13, pp. 3006-3019, 2012.

[22] S. H. Sandilo and W. T. van Horssen, "On variable length induced vibrations of a vertical string," Journal of Sound and Vibration, vol. 333, no. 11, pp. 2432-2449, 2014.

[23] Y. Wang, G. Cao, Z. Zhu, J. Wang, and N. Wang, "Longitudinal response of parallel hoisting system with time-varying rope length," Journal of Vibroengineering, vol. 16, pp. 4088-4101, 2014.

[24] T. W. Park, O. Software Inc, and E. J. Haug, "Hybrid numerical integration method for machine dynamic simulation," Journal of Mechanisms, Transmissions, and Automation in Design, vol. 108, no. 2, pp. 211-216, 1986.

[25] W. D. Zhu and H. Ren, "An accurate spatial discretization and substructure method with application to moving elevator cable-car systems-part I: methodology," Journal of Vibration and Acoustics, vol. 135, no. 5, Article ID 051036, 2013.

[26] F. E. Udwadia and R. E. Kalaba, "The geometry of constrained motion," Journal of Applied Mathematics and Mechanics, vol. 75, no. 8, pp. 637-640, 1995.

[27] W. D. Zhu and G. Y. Xu, "Vibration of elevator cables with small bending stiffness," Journal of Sound and Vibration, vol. 263, no. 3, pp. 679-699, 2003.

[28] J. Wang, G. Cao, Y. Wang, and R. Wu, "A novel driving strategy for dynamic simulation of hoisting rope with timevarying length," International Journal of Modeling, Simulation, and Scientific Computing, vol. 4, no. 3, Article ID 1350009, 2013. 


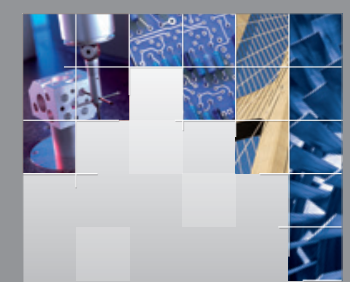

\section{Enfincering}
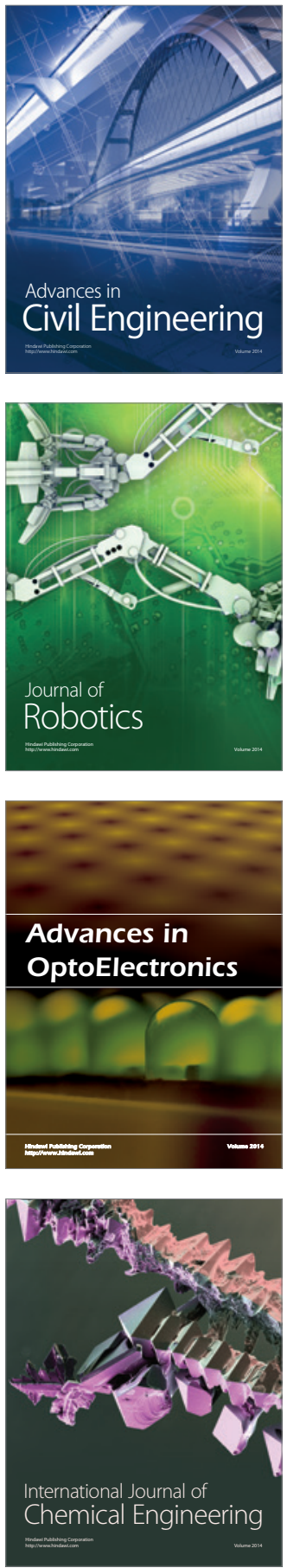

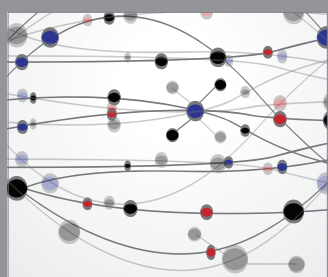

The Scientific World Journal

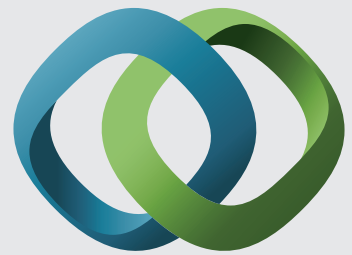

\section{Hindawi}

Submit your manuscripts at

http://www.hindawi.com
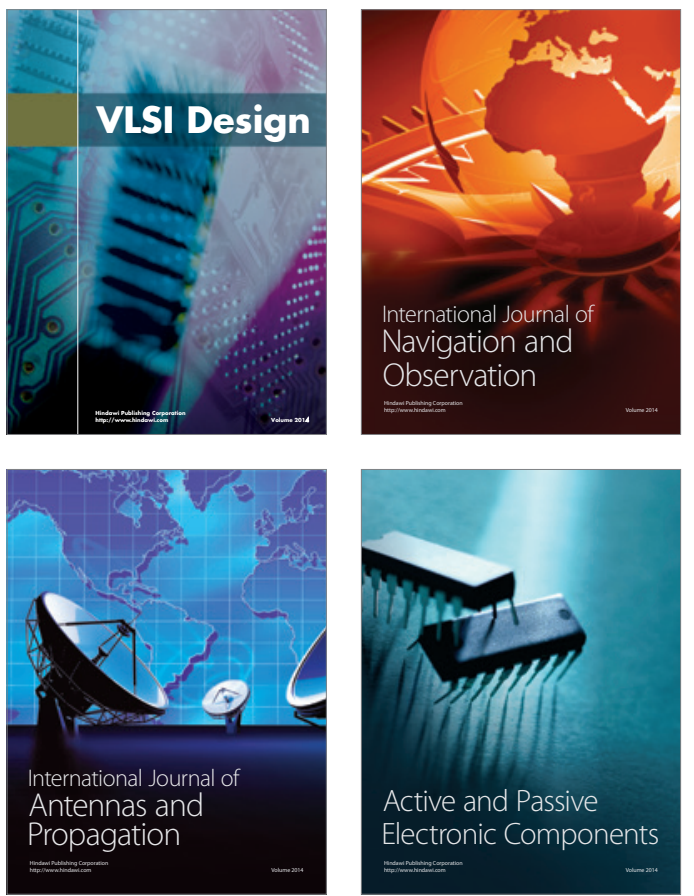
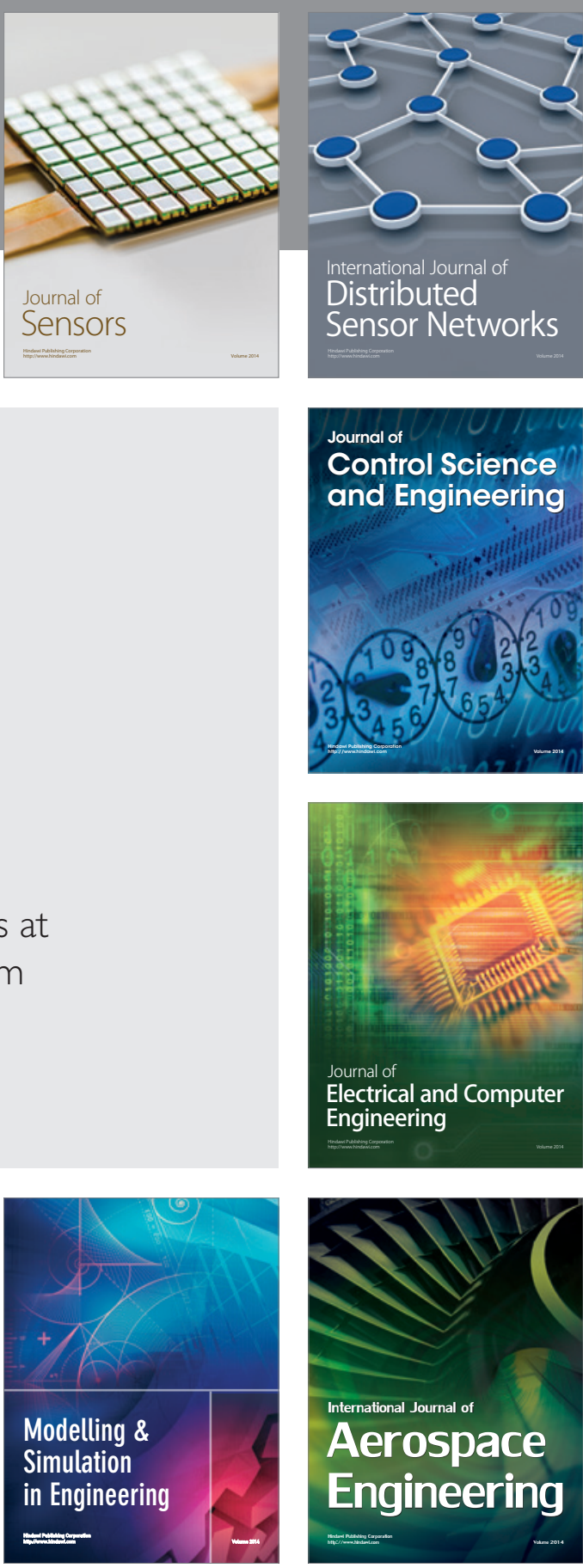

International Journal of

Distributed

Sensor Networks

Journal of

Control Science

and Engineering
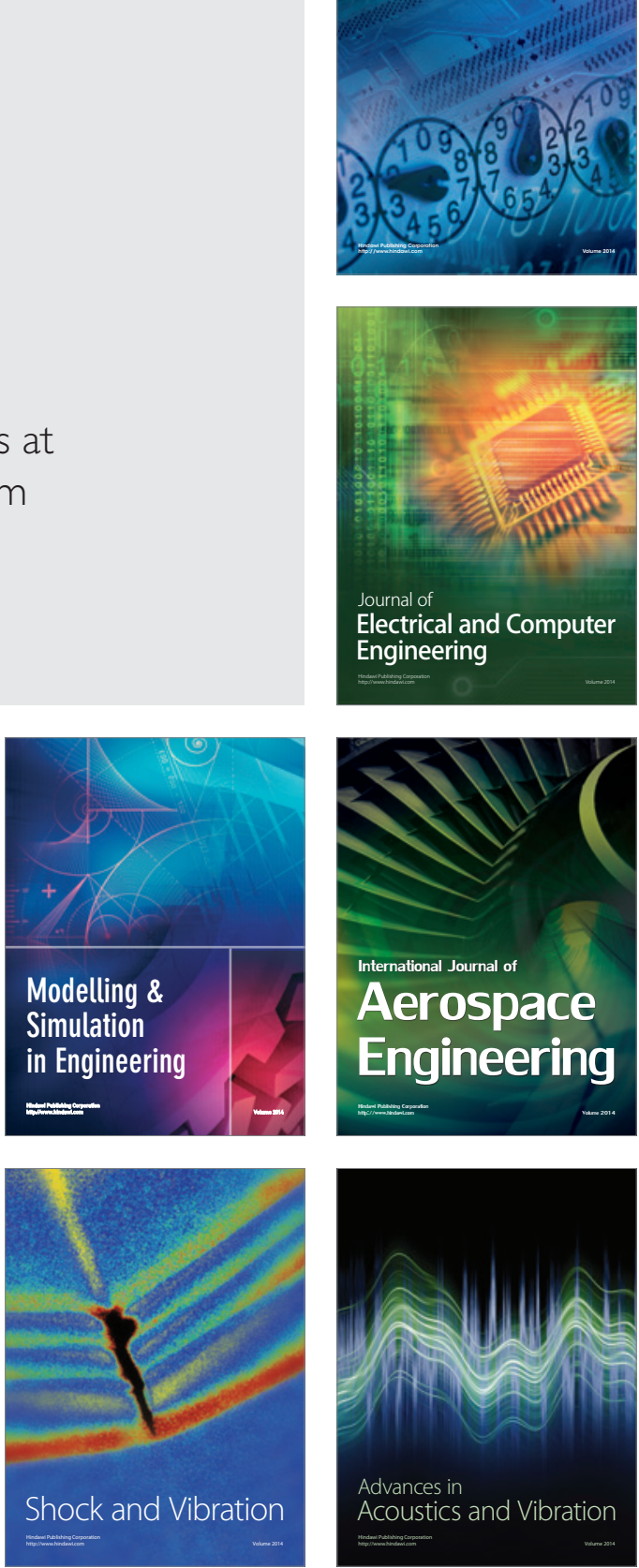